
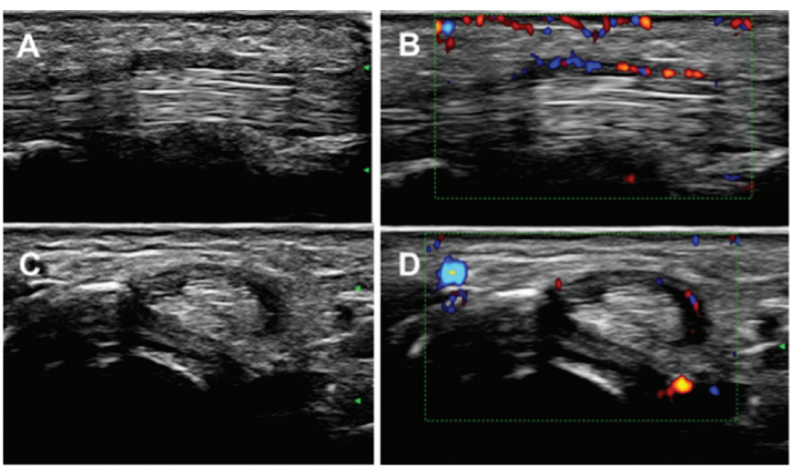

Abstract SAT0306 - Figure 1. The increased thickness and the presence of Doppler signals in the A1 pulley in a digit with dactylitis in longitudinal (A and B) and transverse (C and D)

Results: Half of the A1 pulleys with dactylitic digits had Doppler signals (10/20, $50 \%)$, which was less common in A2 $(6 / 20(30 \%))$ and A4 pulleys $(6 / 20(30 \%))$ (figure). The digits without dactylitis had Doppler signals less frequently (A1: 1/19 (5\%); A2: 1/19 (5\%) A4: 1/19 (5\%))

A1, A2 and A4 pulleys were significantly thicker in dactylitis fingers compared to fingers without dactylitis, both in longitudinal and transverse planes (table 1). Conclusions: This study demonstrates that pulleys contribute to the pathogenesis of dactylitis with increased vascularity and thickening, probably due to the micro-enthesitis at the level where the flexor tendons are exposed to high mechanical stress. This is important to understand the anatomical basis of a complex disease feature in PsA, dactylitis

Disclosure of Interest: None declared

DOI: 10.1136/annrheumdis-2018-eular.7258

\section{SAT0307 IMPACT OF THE MODIFIED RHEUMATIC DISEASE COMORBIDITY INDEX(MRDCI) ON DRUG SURVIVAL OF FIRST LINE ANTI-TNFA DRUGS IN PATENTS AFFECTED WITH PSORIATIC ARTHRITIS IN REAL LIFE SETTING}

M. Fornaro ${ }^{1}$, V. Venerito ${ }^{1}$, L. Cantarini ${ }^{2}$, M.G. Anelli ${ }^{1}$, F. Cacciapaglia ${ }^{1}$

G. Lopalco ${ }^{1}$, G. Lapadula ${ }^{1}$, F. lannone ${ }^{1} .{ }^{1}$ Rheumatology Unit; Department of Emergency and Organs Transplantation (DETO), University of Bari (Italy), BARI; ${ }^{2}$ Research Center of Systemic Autoinflammatory Diseases and Behçet's Disease Clinic, Department of Medical Sciences, Surgery and Neurosciences, Università di Siena, Siena, Italy

Background: Psoriatic Arthritis (PsA) is a chronic condition resulting in significant physical disability and, in many cases, accelerated mortality. Studies have shown that patients with PsA suffer also from associated comorbidities, including cardiovascular diseases, obesity and metabolic syndrome, diabetes, osteoporosis, malignancy and depression; these could also play a role in determining discontinuation from therapy, especially if anti-TNF $\alpha$ drugs are prescribed.

Objectives: Our study's goal is to demonstrate the impact of comorbidities on drug survival of first line anti-TNF $\alpha$ treatment in a cohort of patients affected with PsA.

Methods: We retrospectively assessed 208 patients, (136 female, 72 male) mean age $( \pm S D) 51.35 \pm 12.34$ years, fulfilling the CASPAR criteria for PSA who underwent first line anti-TNF $\alpha$ therapy in two centres for a mean duration of 23.34 \pm 15.50 , from 2011 to 2016 . Disease characteristics were registered at first entry. To evaluate the burden of comorbidities we used the modified Rheumatic Disease Comorbidity Index (mRDCl), ${ }^{1}$ a validated score including lung illnesses, cardiovascular diseases, stroke, hypertension, gastrointestinal disorders, diabetes, fractures, depression, obesity, kidney diseases and cancer. The mRDCI was scored at baseline as well. Drug retention was analysed using Kaplan-Maier curves. Cox regression models were used to estimate the inference of $\mathrm{mRDCl}$ and several disease characteristics on drug discontinuation. Goodness of fit of the final model was assessed comparing Cox-Snell residuals to Nelson-Alen cumulative hazard function.

Results: Drug persitence in first line therapy was significantly higher in patients with $\mathrm{mRDCl}<4(70.43 \%)$ than in those with $\mathrm{mRDCl} \geq 4(45.45 \%)$. Compared to $\mathrm{mRDCl}>4$, those with $\mathrm{mRDCl}<4$ showed significantly higher drug survival rate $(p=0.018)$. Multivariate Cox model showed that $m R D C l \geq 4$ (HR 1.94) and female gender (HR 2.39) were strong predictors of drug discontinuation. Nelson-Alen hazard function followed very closely Cox-Snell residuals showed final model fitted well the data except for large values of time.

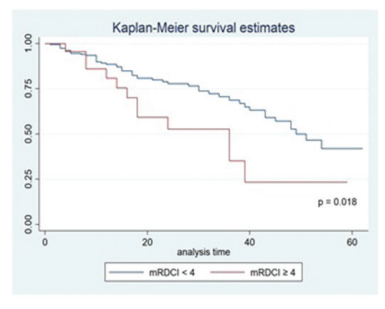

Abstract SAT0307 - Figure 1. Drug survival in first-line anti-TNF $\alpha$ treatment assessed by Kaplan-Meier and comparison among patients with $\mathrm{mRDCl}<4$ and $\mathrm{mRDCl} \geq 4$.

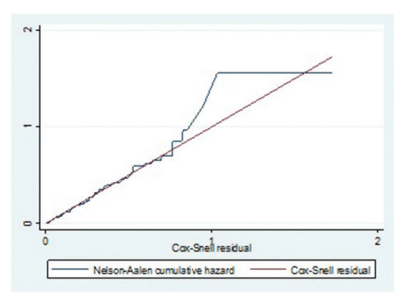

Abstract SAT0307 - Figure 2. Nelson-Alen Hazard function and Cox-Snell residual

Conclusions: This study shows that an high $\mathrm{mRDCl}$ at baseline negatively impacts the persistence on first line anti-TNF $\alpha$ treatment in patients affected with PsA in real life setting; hence Rheumatologists should take into account comorbidities in the management of PsA and in admninistering anti-TNF $\alpha$ therapy as these may condition the persistence on therapy.

\section{REFERENCE:}

[1] Staetgens, et al. Content and construct validity of the Rheumatic Diseases Comorbidity Index in patients with gout. Rheumatology 2015 Sep;54 (9):1659-1663.

Disclosure of Interest: None declared DOI: 10.1136/annrheumdis-2018-eular.2216

\section{SAT0308 PROSPECTIVE OBSERVATIONAL STUDY ON OCULAR INVOLVEMENT IN PATIENTS AFFECTED BY MODERATE TO SEVERE PSORIATIC ARTHRITIS}

C. Canofari ${ }^{1}$, M.S. Chimenti ${ }^{1}$, P. Conigliaro ${ }^{1}$, F. Sunzini ${ }^{1}$, P. Triggianese ${ }^{1}$, G. Draghessi ${ }^{2}$, F. Ambrifi $i^{2}$, A.G. Salandri ${ }^{2}$, M. Cesareo $^{2}$, R. Perricone ${ }^{1}$

${ }^{1}$ Department of System Medicine, Rheumatology, allergology and clinical immunology; ${ }^{2}$ Ophthalmology Unit, Department of Experimental Medicine and Surgery, University of Rome Tor Vergata, Rome, Italy

Background: Psoriatic Arthritis (PsA) is a chronic inflammatory arthritis typically often associated to several comorbidities. The presence of eye involvement in terms of uveitis is well known in PsA patients while retinal abnormalities and dry eye need to be characterised

Objectives: The aim of the study was to analyse subclinical retinal abnormalities and dry eye in a cohort of PsA patients who were naïve to biologic treatments

Methods: In a prospective cross-sectional study, consecutive PsA patients without clinical eye involvement were enrolled (time frame January 2017-December 2017). Joint disease activity and ESR and CRP were measured. Functional and morphological eye assessment included: complete ophthalmological examination, ocular surface disease index (OSDI), Schirmer test, BUT, spectral-domain optical coherence tomography (SD-OCT), standard automated perimetry (SAP, measured as mean defect - MD - and pattern standard deviation - PSD), and fundus perimetry (FP). Data were compared to findings from the eyes of 24 age/sex matched healthy controls $(\mathrm{HC})$.

Results: A total of 58 eyes from 29 PsA patients (21 women and 8 men; age 52.7 \pm 13.3 years) and 48 eyes from $\mathrm{HC}$ ( 14 women and 10 men; age $47.6 \pm 15$ years) were evaluated.

Overall, most of the PsA patients showed a normal Schirmer test, with the exclusion of three patients (10.3\%). PsA patients showed abnormal OSDI in $55.2 \%$ and lower BUT in compared to $\mathrm{HC}(\mathrm{p}<0.001$, Fig.1A). ESR resulted positively correlated with OSDI $(p<0.001, r=0.6$, Fig.1B), and negatively related with Schirmer test $(p=0.005, r=-0.5$, Fig. $1 C)$ and BUT $(p=0.04, r=-0.4$, Fig.1D). In the PsA group, SAP tests showed a lower MD $(p<0.0001)$ and a higher PSD $(p=0.0043)$ in 
comparison with the $\mathrm{HC}$. The latter PSD analysis resulted positively correlated with ESR $(p=0.01 ; r=0.5)$ and CRP values $(p=0.001 ; r=0.4)$. FP mean differential sensitivity and FP mean defect values were lower in PsA patients with respect to $\mathrm{HC}(\mathrm{p}<0.0001$ for both the comparisons, figure $1 \mathrm{E}-\mathrm{F})$ and resulted negatively correlated with the age $(p=0.03 r=-0.4$ for both the correlations, figure $1 \mathrm{G}-\mathrm{H})$. SDOCT in the posterior pole (superior and inferior hemifields) did not reveal differences for the mean retinal thickness between PsA patients and $\mathrm{HC}$
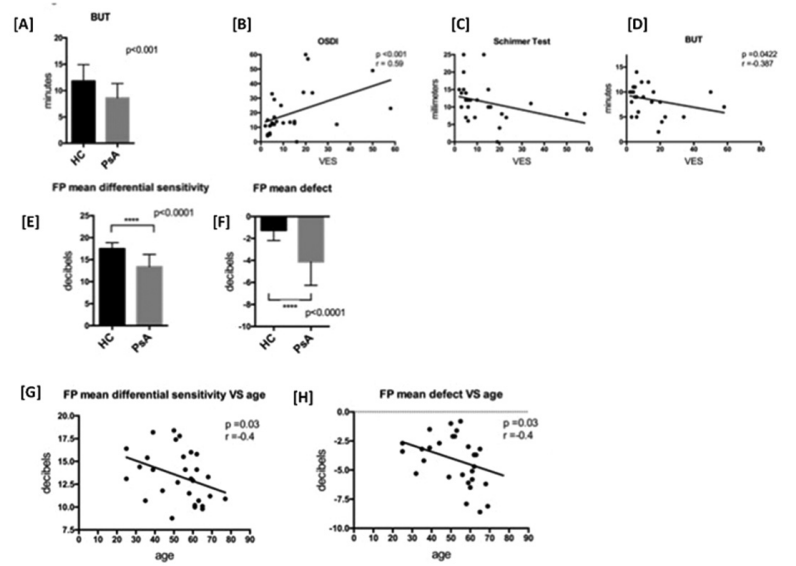

Conclusions: Intriguingly, an impairment in quality of tear film in PsA patients was observed compared to HC. The correlation between ESR and dry eye tests may be explained with a potential relationship between systemic inflammation and sicca syndrome.

Interestingly, PsA patients showed a retinal functional impairment by reduced retinal sensitivity measured by MD, FP mean differential sensitivity and FP mean defect values.

To our knowledge this is the first study investigating eye function and morphology in PsA patients. Further studies are needed to confirm and explain these results. Disclosure of Interest: None declared

DOI: 10.1136/annrheumdis-2018-eular.4554

\section{SAT0309 UNDERESTIMATION OF CARDIOVASCULAR EVENTS BY CARDIOVASCULAR RISK SCORES IN PSORIATIC ARTHRITIS PATIENTS}

H.M. Lam ${ }^{1}$, S.H.O. Ngai ${ }^{1}$, S.H. Cho ${ }^{1}$, T.K. Chun ${ }^{1}$, K.L. Kot ${ }^{1}$, C.T. Yim ${ }^{1}$, E.F. Yu ${ }^{1}$, E. W.L. Kun ${ }^{2}$, L.S. Tam ${ }^{1} .{ }^{1}$ Department of Medicine and Therapeutics, THE CHINESE UNIVERSITY OF HONG KONG, Sha Tin; ${ }^{2}$ Department of Medicine, Alice Ho Miu Ling Nethersole Hospital, Tai Po, Hong Kong

Background: Compared with the general population, patients with Psoriatic Arthritis (PsA) have elevated risks of developing cardiovascular diseases (CVD). The performances of established CVD risk scores in PsA patients have not been fully evaluated yet. European League Against Rheumatism (EULAR) recommends a 1.5 multiplication factor to these CVD risk scores when it is applied in rheumatoid arthritis patients. Whether the same multiplication factor could improve the performance of the risk scores in PsA patients is unknown.

Objectives: To investigate the performances of various CVD risk scores and their EULAR modified versions for predicting CVD events in PsA patients

Methods: Prospectively collected data from the two Hong Kong PsA cohort was used. Discriminatory ability for CV risk prediction was estimated by the area under the receiver operating characteristic curve (AUC). Four different CVD risk scores namely Framingham risk score (FRS), QRISK II, HeartScore and American College of Cardiology and American Heart Association (ACC/AHA) 10 year atherosclerotic cardiovascular disease (ASCVD) and their EULAR modified versions were calculated at baseline. The primary outcome was first CVD events, including stable and unstable angina, myocardial infarction, ischaemic and haemorrhagic stroke, transient ischaemic attack, heart failure, coronary insufficiency, pericardial disease, peripheral arterial disease, thrombosis, percutaneous coronary intervention (angioplasty), coronary artery bypass graft, implantation of pacemaker or defibrillator and CVD death.

Results: 228 patients [48.9 \pm 11.8 years, male: $124(54.4 \%)]$ were recruited between 2006 to 2016. Baseline data were available from 227, 226, 226 and 188 patients to calculate the FRS, QRISKII, HeartScore and ASCVD, respectively. After a mean follow up of $6.7 \pm 4.7$ years, 30 patients $(13.2 \%)$ experienced a CVD event (CVD +group). At baseline, the CVD + group was significantly older $(57.8$ \pm 12.0 vs $47.6 \pm 11.2$ years; $p<0.001)$, with a higher prevalence of diabetes $(26 \%$ vs $12 \% ; p<0.021$ ), had higher systolic blood pressure (SBP: $142 \pm 22.0$ vs 128 $\pm 19.6 \mathrm{mmHg} ; \mathrm{p}<0.001$ ) and higher triglycerides (TG: $1.9 \pm 1.3$ vs $1.4 \pm 0.8 \mathrm{mmol} / \mathrm{L}$, $\mathrm{p}=0.027$ ). All CVD risk scores were significantly higher in the CVD +group (FRS: $18.3 \pm 13.1$ vs $8.9 \pm 8.7, p<0.001$; QRISKII: $11.9 \pm 8.6$ vs $4.9 \pm 5.0, p<0.001$; HeartScore: $2.3 \pm 2.1$ vs $0.9 \pm 1.3, p=0.001$; ASCVD: $14.5 \pm 12.8$ vs $4.8 \pm 5.2, p=0.001$ ) AUC for FRS, QRISKII, HeartScore and ASCVD were $0.74(0.64-0.83, p<0.001)$ $0.76(0.66-0.86, \mathrm{p}<0.001), 0.72(0.62-0.83, \mathrm{p}<0.001)$, and $0.77(0.67-0.86$ $\mathrm{p}<0.001)$, respectively. In total, $76(33.5 \%), 9(4.0 \%), 7(3.1 \%)$ and $47(25.0 \%)$ patients were classified as high CVD risk according to FRS $>10 \%$, QRISK $\|>20 \%$, HeartScore $>5 \%$ and ASCVD $>7.5 \%$ respectively. In the CVD +group, those identified as high risk were only $63 \%$ (by FRS), 20\% (by QRISK2), 13.3\% (by HeartScore) and 46\% (by ASCVD) (figure1a). By applying the EULAR multiplication factor, $80 \%, 36 \%, 26.67 \%$ and $56.7 \%$ of the patients with CVD +were reclassified as high risk (figure $1 \mathrm{~b}$ ).

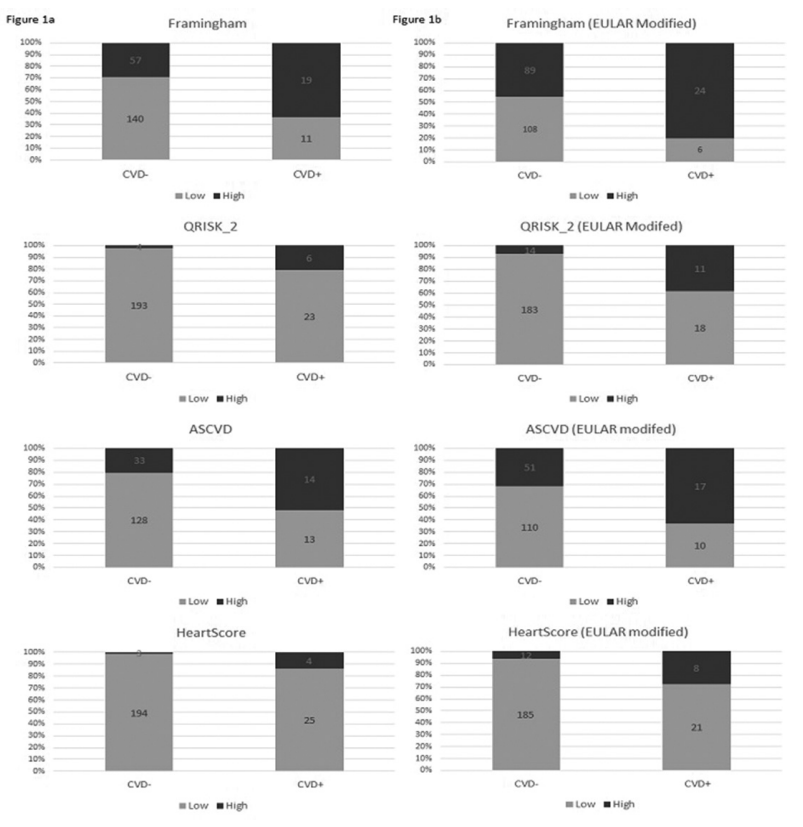

Abstract SAT0309 - Figure 1

Conclusions: All CVD risk scores significantly underestimated CVD risks among PsA patients. This study demonstrated for the first time that adaptation of the EULAR recommendation only improved the accuracy of FRS to a moderate level. Disclosure of Interest: None declared

DOI: 10.1136/annrheumdis-2018-eular.6074

\section{SAT0310 THE ASSOCIATIONS OF SERUM IL18 AND OSTEOPROTEGERIN (OPG) LEVELS WITH THE LIPID PROFILE IN PSORIATIC ARTHRITIS (PSA) PATIENTS}

K. Bonek ${ }^{1}$, P. Gluszko ${ }^{1}$, E. Kontny ${ }^{2} .{ }^{1}$ Department of Rheumatology; ${ }^{2}$ Department of Pathophysiology and Immunology, National Institute of Geriatrics, Rheumatology and Rehabilitation, Warszawa, Poland

Background: We have previously found that IL-18 and OPG serum concentrations are correlated with cardiovascular (CV) risk in psoriatic arthritis but not in ankylosing spondylitis (AS) patients. ${ }^{1}$

Objectives: To investigate whether in PsA patients the association of OPG and IL-18 with CV risk is mediated by an impact of these cytokines on lipid profile changes.

Methods: 49 patients with PsA (25 M/24 F) with $(n=10)$ and without $(n=39)$ coronary heart disease (CHD), and 25 sex and age matched (mean age 44,4 vs 43,4 years) patients with AS were enrolled. Disease activity was measured by DAPSA $(25,17 \pm 19,9)$ in PsA group and by BASDAI $(5,37 \pm 2)$ and ASDAS- CRP $(3,18 \pm 1)$ in AS group. The lipid profile (triglycerides - TG, total cholesterol - tChol, low- and high-density lipoprotein - LDL and HDL, respectively), systemic inflammation markers and cytokines (OPG, IL-18) were measured in patients serum samples Atherogenic index $(\mathrm{Al}=\mathrm{tChol} / \mathrm{HDL})$ was calculated. Statistical analysis was performed using Mann-Whitney U-test and Spearman's Rank test. Data are expressed as mean values.

Results: Patients with PsA presented more atherogenic lipid profile than AS patients because of their higher TG levels (153 vs $126,6 \mathrm{mg} / \mathrm{dl} ; \mathrm{p}=0,05)$ and $\mathrm{Al}$ values $(3,83$ vs 3,$24 ; p=0,05)$ while lower HDL concentrations $(51,6$ vs $61,4 \mathrm{mg} /$ 